\title{
Cloud inhomogeneity and broadband solar fluxes
}

\author{
R. Scheirer ${ }^{1}$ and A. Macke \\ Department of Ocean Circulation and Climate, Institute for Marine Research, Kiel, Germany
}

Received 16 December 2002; revised 27 June 2003; accepted 14 July 2003; published 7 October 2003.

[1] Simplified representations of spatially inhomogeneous (three-dimensional (3-D)) clouds in radiative transfer models provide systematic errors when calculating solar broadband radiative fluxes. An example is the neglect of horizontal photon transports as it is the case for the independent column approximation (ICA). The present work tries to quantify and interpret these errors on the basis of a large set of 3-D mixed phase cloud scenarios with 3-D varying extinction coefficients, scattering phase functions, and single-scattering albedos. The cloud cases result from a mesoscale atmospheric circulation model with detailed cloud microphysics. Domain-averaged cloud radiative fluxes are calculated by means of a Monte Carlo radiative transfer model. Depending on cloud type and solar zenith angle (SZA) the differences between 3-D and ICA results range from $+20 \mathrm{~W} \mathrm{~m}^{-2}$ to $-30 \mathrm{~W} \mathrm{~m}^{-2}$ for the upward reflected fluxes and from $+10 \mathrm{~W} \mathrm{~m}^{-2}$ to $-7 \mathrm{~W}$ $\mathrm{m}^{-2}$ for the absorbed fluxes. The mean (averaged over all cloud realizations) errors of the ICA-based upward fluxes vary between $5 \mathrm{~W} \mathrm{~m}^{-2}$ overestimation at $15^{\circ} \mathrm{SZA}$ and $6 \mathrm{~W} \mathrm{~m}^{-2}$ underestimation at $75^{\circ} \mathrm{SZA}$. The ICA underestimates the absorbed flux by $\sim 1-$ $2 \mathrm{~W} \mathrm{~m}^{-2}$ for most SZA except for $75^{\circ}$. It is found that neglecting the horizontal variability of the absorption and scattering properties of the cloud hydrometeors leads to a general underestimation of solar broadband absorption by as much as $15 \mathrm{~W} \mathrm{~m}^{-2}$ with average values between $4 \mathrm{~W} \mathrm{~m}^{-2}$ at small SZA and $1 \mathrm{~W} \mathrm{~m}^{-2}$ at large SZA. INDEX TERMS: 0669 Electromagnetics: Scattering and diffraction; 3359 Meteorology and Atmospheric Dynamics: Radiative processes; 3360 Meteorology and Atmospheric Dynamics: Remote sensing; KEYWORDS: cloud inhomogeneity, solar flux, anomalous absorption

Citation: Scheirer, R., and A. Macke, Cloud inhomogeneity and broadband solar fluxes, J. Geophys. Res., 108(D19), 4599, doi:10.1029/2002JD003321, 2003.

\section{Introduction}

[2] The solar radiative fluxes of atmospheric clouds are strongly affected by the cloud spatial structure. In most cases this structure is not fully considered in radiative transfer calculations partly because of insufficient information about the cloud structure and partly because of the high computational costs.

[3] Since it is well established that representing a specific cloud by a single horizontally homogeneous (one-dimensional (1-D)) geometry leads to significant errors in calculating the solar cloud radiative fluxes [e.g., Cahalan et al., 1994] the Independent Column Approximation (ICA) has become the focus of much attention. Here the 3-D clouds are treated as independent superpositions of individual horizontally plane parallel columns. The advantage of the ICA is that classical 1-D radiative transfer tools can be applied to 3-D cloud fields. In comparison to the correct 3-D solution the ICA neglects horizontal transports only and is often regarded as a sufficiently accurate solution to the 3-D radiative transfer problem for domain-averaged radiative

\footnotetext{
${ }^{1}$ Now at the Institut für Physik der Atmosphäre, German Aerospace Center, Oberpfaffenhofen, Germany
}

Copyright 2003 by the American Geophysical Union. 0148-0227/03/2002JD003321 fluxes. Therefore the main focus of this study is to quantify and to physically explain the errors of the ICA.

[4] Benner and Evans [2001] have performed a similar error analysis of the ICA and other radiative transfer approximations. Their work investigated small tropical cumulus (optical thickness around 4) as resulting from shallow convection. Only little deviations of the various approximations compared to the 3-D results were found. However, the present work will demonstrate that accounting for optically thicker cloud systems will lead to more pronounced horizontal photon propagations with corresponding significant errors of the ICA.

[5] It is still discussed, if and to what extent the atmospheric absorption is systematically underestimated due to simplified radiative transfer modeling in large scale atmospheric circulation models, (catchword "cloud anomalous absorption" [e.g., Cess et al., 1995; Pilewskie and Valero, 1995]). In this context we propose a simple mechanism that explains a general increase of absorption if horizontal variations of cloud particle size are accounted for.

[6] The problem of 3-D radiative transfer and its various aspects in energetic and remote sensing issues has been extensively studied by many authors. While the first simulations of 3-D radiative transfer in the cloudy atmosphere were essentially based on artificial cloud constructions (mostly bounded cascade models [e.g., Marshak et al., 1994]), present 


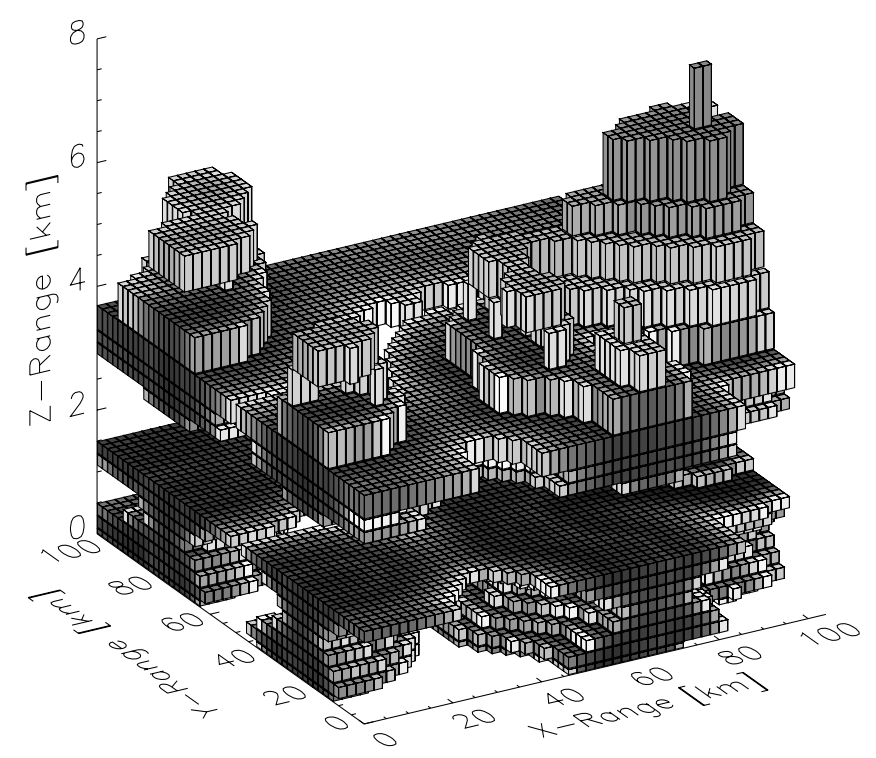

Figure 1. Example of an inhomogeneous multilayered cloud consisting of liquid water, ice, rain, and snow. Solid boxes denote large extinction coefficients.

work uses more and more cloud fields from small-scale cloudresolving atmospheric models. However, most applications have been limited to pure water clouds [e.g., O'Hirok and Gautier, 1998] or to few cloud realizations [e.g., Barker, 1996; Barker et al., 1999; Fu et al., 2000] with simplified treatments of cloud microphysical properties. The present work tries to extend these studies to (1) a considerably larger set of 3-D cloud realizations and (2) to clouds with detailed 3 -D scattering and absorption processes of the different types of hydrometeors (water droplets, ice, rain, snow) as they occur in many mixed-phase clouds. In contrast to most other studies on this subject, the present work accounts for the full 3-D nature of the cloudy atmosphere. It will be shown that this second point explains a systematic enhancement in the solar cloud absorption by as much as $15 \mathrm{~W} \mathrm{~m}^{-2}$.

[7] The microphysical and macrophysical properties of the 3-D clouds used in this study are described in section 2. Section 3 specifies how scattering and absorption at atmospheric gases and hydrometeors is taken into account. The Monte Carlo radiative transfer model is described in section 4, followed by a presentation and discussion of the results in section 5. Section 6 provides a summary and conclusion.

\section{Clouds}

[8] The spatial cloud fields under investigation are calculated by means of the three-dimensional (3-D), nonhydrostatic mesoscale atmospheric model (GESIMA) [Eppel et al., 1998; Hagedorn, 1996].

[9] Cloud microphysical processes in GESIMA are based on a bulk parameterization by Levkov et al. [1992]. The spatially resolved GESIMA data that are required for the radiative transfer calculations are temperature, pressure, water vapor content, water content for liquid water, snow, ice, and rain, as well as the number concentrations of these hydrometeors. These data are transformed to volume extinction coefficients and effective particle sizes as described by Macke et al. [1999].
[10] Cloud developement in GESIMA calculations is forced by adding a local water vapor excess in each vertical layer at 11 horizontally randomly choosen subdomains. Grid cells with initially largest relative humidity obtain the largest supply of water vapor resulting in a local maximum increase of relative humidity of up to $10 \%$. This leads to occasional supersaturation which in turn triggers local cumulus convection. The water vapor supply continues for $50 \mathrm{~min}$, and on average, $6 \%$ of the initial water vapor amount has been added in each layer. The cloud model runs are stopped after 2 hours. See von Bremen et al. [2002] for details regarding the cloud generation. The resulting cloud structure (convective, stratiform, multilayered) mainly depends on the initial humidity and temperature profile and has been identified by visual inspection. Out of 133 cloud realizations, 46 are stratiform, 50 are convective, and 37 have separate layers (e.g., Figure 1). For a short overview of the different cloud types and their geometrical properties, see Table 1.

[11] For the Monte Carlo radiative transfer calculations the model domain is reduced to the smallest right parallelepiped that embraces cloud top and cloud sides. Upward directed flux is calculated at the top of the cloud, i.e., at the highest layer which still contains a cloudy grid box. The lower bound of the model domain is set to the ground surface where also downward transmitted flux is calculated. The absorbed flux is related to the entire model domain.

\subsection{Stratiform and Convective Clouds}

[12] The GESIMA cloud realizations for stratiform and convective clouds are initialized with five temperature and humidity profiles taken from radiosonde measurements over the Baltic Sea area during summer 1995 and 1996, over the South Pacific in February 1995, and over the North Atlantic during January and July 1989 [Hagedorn, 1996]. The model runs for roughly 2 hours with a time step of $10 \mathrm{~s}$. Every 5 minutes a cloud realization is taken from the model run so that a maximum of 25 cloud realizations result from each

Table 1. Statistics of the Used Cloud Fields for the Cloud Top, Cloud Bottom, Geometric Thickness, and Cloud Cover ${ }^{\mathrm{a}}$

\begin{tabular}{lccc}
\hline & Convective & Stratiform & Multilayered \\
\hline & & Cloud Top & \\
Min. & $2500 \mathrm{~m}$ & $2500 \mathrm{~m}$ & $2300 \mathrm{~m}$ \\
Mean & $5182 \mathrm{~m}$ & $5241 \mathrm{~m}$ & $5138 \mathrm{~m}$ \\
Max. & $8000 \mathrm{~m}$ & $8000 \mathrm{~m}$ & $9000 \mathrm{~m}$ \\
& & & \\
Min. & $100 \mathrm{~m}$ & $100 \mathrm{~m}$ & $0 \mathrm{~m}$ \\
Mean & $836 \mathrm{~m}$ & $1343 \mathrm{~m}$ & $0 \mathrm{~m}$ \\
Max. & $3300 \mathrm{~m}$ & $2700 \mathrm{~m}$ & $0 \mathrm{~m}$ \\
& & & \\
Min. & $1200 \mathrm{~m}$ & $2000 \mathrm{~m}$ & $2300 \mathrm{~m}$ \\
Mean & $4346 \mathrm{~m}$ & $3898 \mathrm{~m}$ & $5138 \mathrm{~m}$ \\
Max. & $6700 \mathrm{~m}$ & $5700 \mathrm{~m}$ & $9000 \mathrm{~m}$ \\
& & & 0.44 \\
Min. & 0.54 & Cloud Cover & 0.89 \\
Mean & 0.70 & 1.0 & 1.0 \\
Max. & 0.87 & 1.0 & 1.0 \\
\hline
\end{tabular}

${ }^{\text {a }}$ Cloud geometric thickness denotes the difference between upper and lower cloud border (for multilayered clouds, the difference between the upper border of the upper layer and the lower border of the lowest layer). Min., minimum; max., maximum. 
Table 2. Names, Wavelength Ranges, Solar Irradiation, Weighting Factors, and Imaginary Part of the Complex Refractive Index of Water for Each Solar Spectral Band Calculated From the Data Set Given by Thekaekara [1974]

\begin{tabular}{ccccc}
\hline Name & Range, $\mu \mathrm{m}$ & Irradiation, $\mathrm{W} \mathrm{m}^{-2}$ & Weight & $\operatorname{Im}(\mathrm{n})$ \\
\hline A & $0.2-0.3927$ & 98.2 & 0.072 & $1.60 \mathrm{E}-08$ \\
B & $0.3927-0.72$ & 567.2 & 0.417 & $1.96 \mathrm{E}-09$ \\
C & $0.72-1.185$ & 402.9 & 0.296 & $4.180 \mathrm{E}-07$ \\
D & $1.185-1.7335$ & 178.1 & 0.131 & $3.03-04$ \\
E & $1.7335-1.9659$ & 36.0 & 0.026 & $1.25-04$ \\
F & $1.9659-2.21294$ & 24.9 & 0.018 & $1.10 \mathrm{E}-03$ \\
G & $2.21294-2.54$ & 20.6 & 0.015 & $6.87 \mathrm{E}-04$ \\
H & $2.54-2.79$ & 10.4 & 0.008 & $2.34 \mathrm{E}-02$ \\
I & $2.79-2.9725$ & 5.6 & 0.004 & 0.2738 \\
J & $2.9725-3.145$ & 4.2 & 0.003 & 0.2096 \\
K & $3.145-3.31$ & 3.3 & 0.002 & $7.53 \mathrm{E}-02$ \\
L & $3.31-3.64505$ & 5.1 & 0.004 & $1.95 \mathrm{E}-02$ \\
M & $3.64505-4.0$ & 3.8 & 0.003 & $3.76 \mathrm{E}-03$ \\
\hline
\end{tabular}

profile. The model domain ranges from the ground to $10 \mathrm{~km}$ height vertically and covers $21 \mathrm{~km} \times 21 \mathrm{~km}$ horizontally. The vertical resolution varies from $100 \mathrm{~m}$ at the ground to $1 \mathrm{~km}$ at the top. The horizontal resolution is $1 \mathrm{~km}$.

\subsection{Multilayer Clouds}

[13] Clouds with separated layers are produced by initializing GESIMA with four radiosonde measurements obtained between $50^{\circ}$ and $70^{\circ} \mathrm{N}$ during July and August 1989. A model integration time of $\sim 2$ hours and a time step (between taken clouds) of $10 \mathrm{~min}$ provide up to 13 cloud realizations for each radiosonde profile. The spatial dimensions are $104 \mathrm{~km} \times$ $104 \mathrm{~km}$ horizontally and $10 \mathrm{~km}$ vertically with a horizontal resolution of $2 \mathrm{~km}$ and the same vertical resolution as for the stratiform and the convective clouds.

\section{Radiative Properties of Atmospheric Gas and Hydrometeors}

[14] Absorption by gas molecules (water vapor and oxygen) is expressed in terms of their single-scattering albedo $\left(\omega_{0}\right)$. Depending on the mean profiles of temperature, pressure, and absorber amount for each of the nine cloud series described above, the profiles of effective transmittance with regard to the gas absorption are calculated for the spectral intervals listed in Table 2. Molecular spectral line data are taken from the High-Resolution Transmission Molecular Absorption Database (HITRAN) [Rothman et al., 1987] and are applied to a band model developed by Scheirer and Macke [2000] to obtain the transmittance for each spectral band given in Table 2 . Transmittances are transformed into molecular absorption coefficients by taking the vertical depth and molecular number density of each model layer into account. Together with the Rayleigh scattering coefficient the single-scattering albedo can then readily be obtained. A more detailed description of this procedure is given by Scheirer and Macke [2000]. The inhomogeneous distribution of the absorption coefficient is realized by multiplying the absorber density of each GESIMA grid box with the molecular absorption coefficient from the precalculated profile.

[15] The Rayleigh scattering cross section and phase function are obtained from an analytic formula given by Bucholtz [1995] and Chandrasekhar [1960], respectively.
The wavelength dependent depolarization factor needed for the correction term in this phase function is fitted to the data given by Bucholtz [1995].

[16] Absorption and scattering properties of cloud particles are obtained from Mie calculations for water droplets and from ray-tracing calculations for nonspherical snow, rain, and ice particles [Macke et al., 1999]. The geometry of raindrops is approximated by Chebychef polynomials [Macke and Grossklaus 1998] with size-dependent polynomial coefficients. For snow and ice particles the geometry of the fractal polycrystal [Macke et al., 1996] was chosen to account for the high irregular nature of these particle types. The ray-tracing results are given with $1^{\circ}$ resolution in scattering angle except for forward and backward scattering where $0.25^{\circ}$ resolution are used.

[17] The total phase function for each grid box is obtained by averaging the Rayleigh scattering phase function and the scattering phase function of the hydrometeors weighted by their individual scattering cross sections. Similarly, the total single-scattering albedo is obtained from the sum of the Rayleigh scattering coefficient and the scattering coefficient of the hydrometeors divided by the total extinction coefficient.

[18] The procedure outlined above results in cloud fields that have pronounced 3-D structures not only in the extinction coefficient respectively optical thickness but also in the absorption and scattering properties of the hydrometeors. In other words, each model grid box has its individual extinction coefficient, scattering phase function, and single-scattering albedo.

\section{Monte Carlo Radiative Transfer}

[19] In order to obtain the solar broadband upwelling and downwelling radiative fluxes, a Monte Carlo radiative transfer model (GRIMALDI, see http:/www.ifm.unikiel.de/fb/fb1/me/research/Projekte/RemSens/SourceCodes/ codes.html), based on the model by Macke et al. [1997], has been applied to the cloud fields.

[20] The influence of different cloud aspect ratios (ratio of cloud vertical to horizontal dimension) is minimized by applying horizontally periodic boundary conditions to the photon paths. The entry point for each incoming photon is randomly distributed along the upper boundary of the model domain. Clouds are illuminated at a fixed solar azimuth angle. The surface is assumed to be black.

[21] Each model grid box is characterized by its position, geometrical dimensions, extinction coefficient, scattering phase function, and single-scattering albedo.

[22] Photons are traced with a direct Monte Carlo simulation as described by Macke et al. [1999]. Absorption is taken into account by multiplying the initial photon weight with the total single-scattering albedo for each scattering event. Sensitivity studies have shown that $2 \times 10^{6}$ photons suffice to obtain statistically stable results for domainaveraged radiative fluxes.

[23] The radiative transfer calculations have been performed for five solar zenith angles (SZA) $\left(15^{\circ}, 30^{\circ}, 45^{\circ}, 60^{\circ}\right.$, and $75^{\circ}$ ) and for the following two cloud geometries: (1) 3-D inhomogeneous clouds with cyclic horizontal boundary conditions (3-D); (2) column-by-column horizontally homogeneous clouds (independent column approximation (ICA)). 

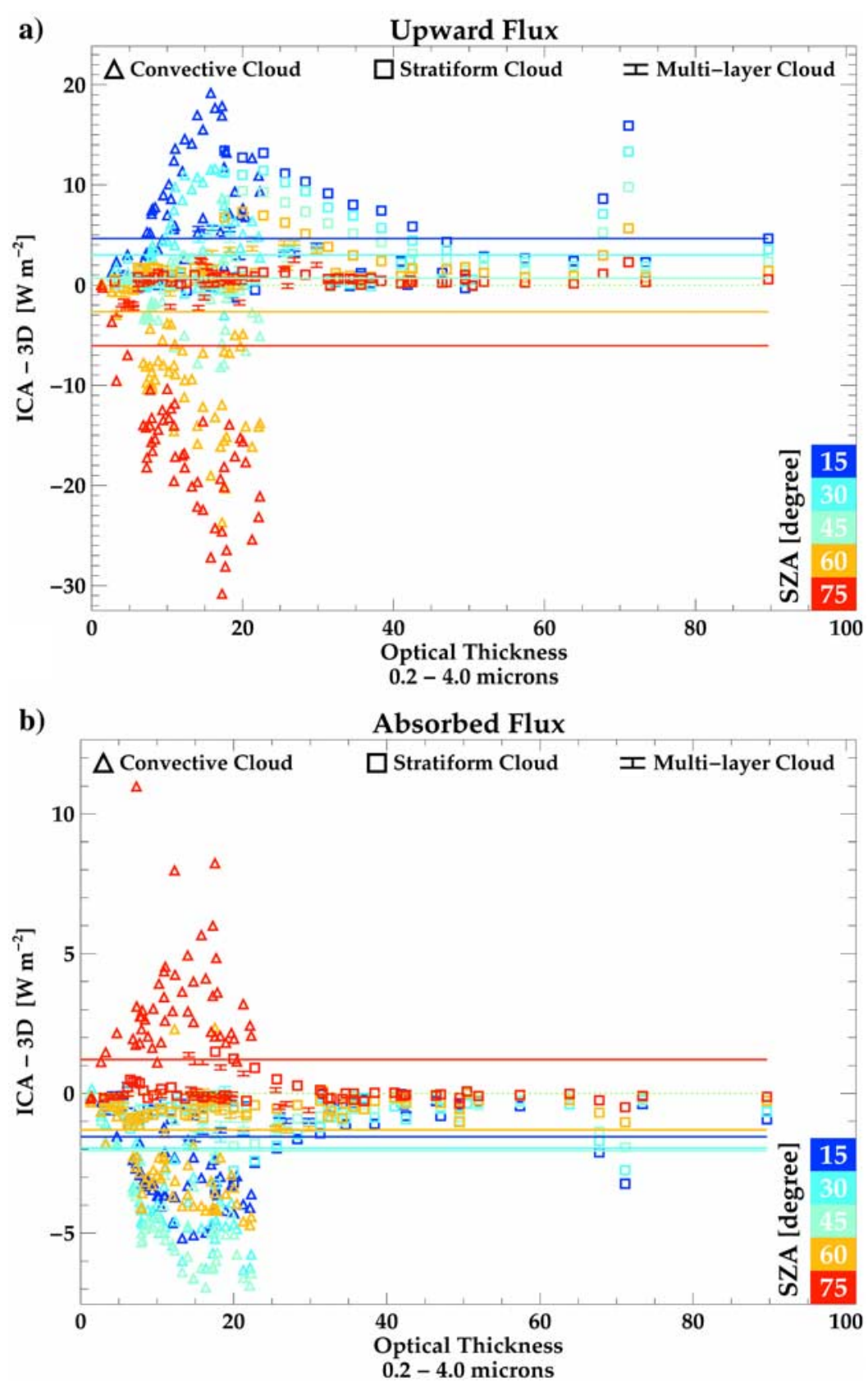

Figure 2. Comparison between independent column approximation (ICA) and three-dimensional (3-D) scenarios. (a) Broadband upward flux and (b) broadband absorption. See text for details.

[24] This results in a total of 1330 experiments for each spectral band (133 clouds, 5 SZA, and 2 different cases (3-D and ICA)). On a Digital Equipment Corporation Alpha workstation (AXP533 AU2) the calculation of $2 \times 10^{6}$ photons ranges from 4 min to 90 min depending on cloud optical thickness and SZA.

\section{Results and Discussion}

[25] Differences in the domain-averaged solar radiative fluxes between the ICA and the 3-D case as a function of cloud optical thickness, solar zenith angle, and cloud type are presented in Figures 2 and 3. Figure 2 shows broadband flux differences, and Figure 3 shows the spectral distributions of mean and standard deviations of absolute (Figures $3 a$ and $3 b$ ) and relative flux differences (Figures $3 \mathrm{c}$ and $3 \mathrm{~d}$ ), i.e., reflected and absorbed fluxes in Figures $3 \mathrm{a}$ and $3 \mathrm{~b}$ and albedo and absorptance in Figures $3 \mathrm{c}$ and $3 \mathrm{~d}$. The terms "error," "underestimation," and "overestimation" used in the following are defined with respect to the 3-D results. Note that in the domain averaging of the optical thickness cloud free columns are included. This results in rather small values in particular for the convective clouds, which have the largest portion of cloud holes (see Table 1) although the individual cloudy columns are optically very thick.

[26] The errors in the upward flux range from $-30 \mathrm{~W} \mathrm{~m}^{-2}$ to $+20 \mathrm{~W} \mathrm{~m}^{-2}$. The mean (averaged over all cloud realizations) differences range from $-6 \mathrm{~W} \mathrm{~m}^{2}$ at a SZA of $75^{\circ}$ to 

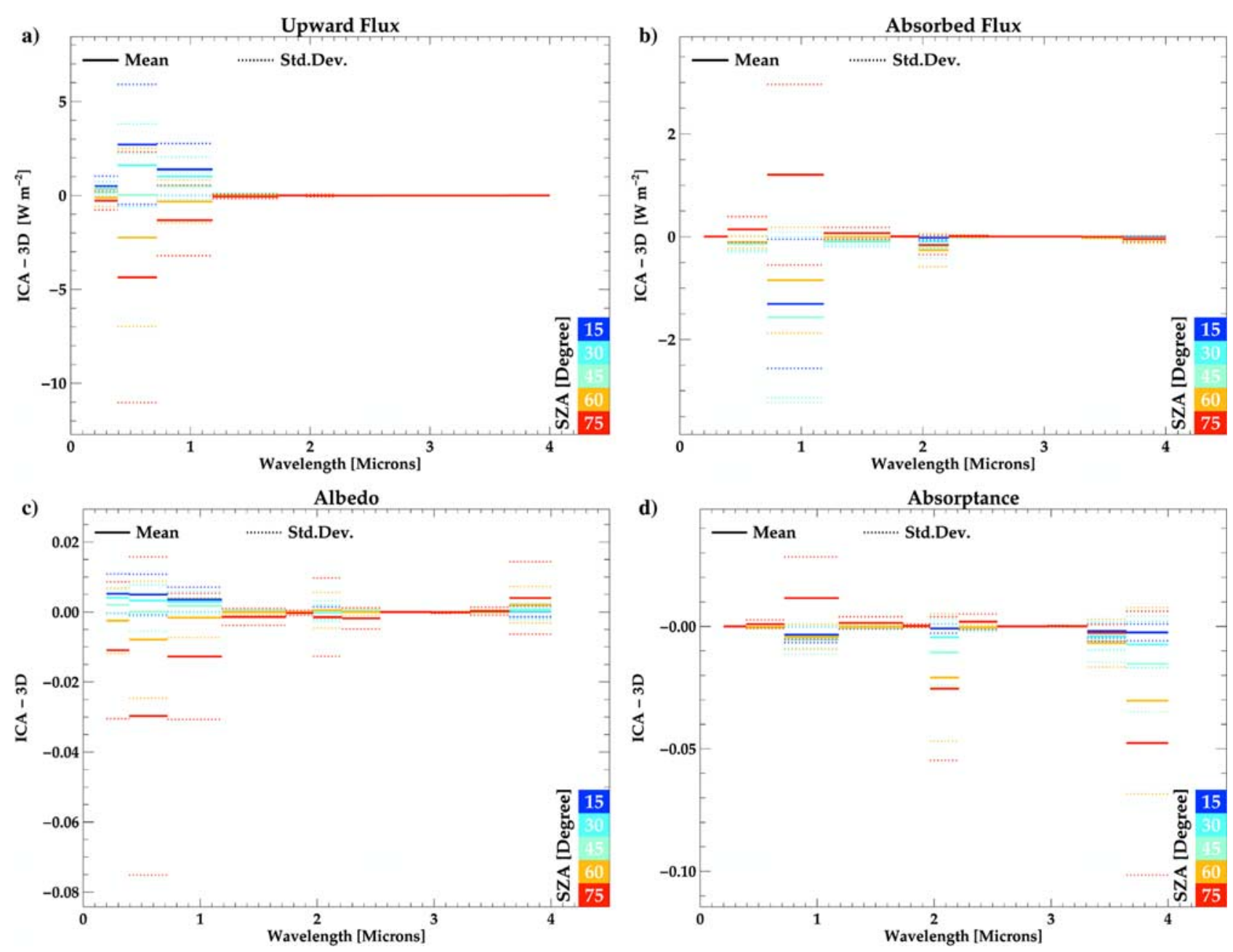

Figure 3. Comparison between ICA and 3-D scenarios. (a, c) Spectral differences in upward fluxes and $(b, d)$ differences in atmospheric absorption. Figures $3 a$ and $3 b$ are spectral fluxes, and Figures $3 \mathrm{c}$ and $3 d$ are spectral intensities. See text for details.

$5 \mathrm{~W} \mathrm{~m}^{-2}$ at $15^{\circ} \mathrm{SZA}$. The differences between ICA and 3-D in atmospheric absorption range from $-7 \mathrm{~W} \mathrm{~m}^{-2}$ at SZA of $45^{\circ}$ to $+11 \mathrm{~W} \mathrm{~m}^{-2}$ at $75^{\circ} \mathrm{SZA}$.

[27] Largest albedo and absorption errors are found for convective clouds as well as for a collection of stratified clouds, which can be referred to as cirrostrati. This cloud type mainly consists of solid ice particles which provide, for the same optical thickness, a larger backscattering and thus larger albedo than spherical water droplets. Though being stratiform, there is a large variability in cloud microphysical properties. This explains the large errors. At low SZA, albedo is overestimated mainly because of an underestimated transmittance. Here the 3-D radiative transfer leads to a net horizontal transport from optically thick into optically thin regions [see Scheirer and Macke, 2001, Figure 5]. This implies a deeper photon penetration which in turn leads to larger transmittance and/or larger absorption. Thus neglecting horizontal transports as it is the case in the ICA leads to smaller transmittance and absorptance and consequently to larger reflection for low SZA. Comparing Figures $3 a$ and $2 b$ shows that the transmission effect is considerably stronger than the absorption effect for the smallest SZA under consideration. At large SZA, 3-D radiative transfer provides net horizontal transports from optically thin into optically thick regions [see again Scheirer and Macke, 2001, Figure 5]. We found for the ICA an overestimation of mean free path length and the number of photon-molecule interactions (not shown here). This is a hint that photons having entered a column of large optical thickness get trapped there, while passing almost undisturbed cloud-free columns. In the 3-D case the lateral incidence into clouds is restricted to the upper boxes (especially at large SZA) where upward reflection is most likely. Thus net horizontal transport at large SZA increases reflection at the expense of absorption and transmission.

[28] Largest underestimations of absorption are found for the moderate SZA of $45^{\circ}$. This appears surprising because there is no extremum in the net horizontal transport for this solar illumination condition. The reason for this is that the deep photon penetration into the cloud in the 3-D case for small SZA essentially leads to large transmittances and does not effect absorption much. Only as the horizontal component of the incoming photon direction increases, the deeper penetrated photons start to get more efficiently absorbed inside the cloud.

[29] The spectral differences in absorption and albedo are shown in. The largest underestimations in albedo (Figure 3c) are found in the visible spectral range and reach $\sim 15 \%$. This is equivalent to $22 \mathrm{~W} \mathrm{~m}^{-2}$ in the upward reflected flux 

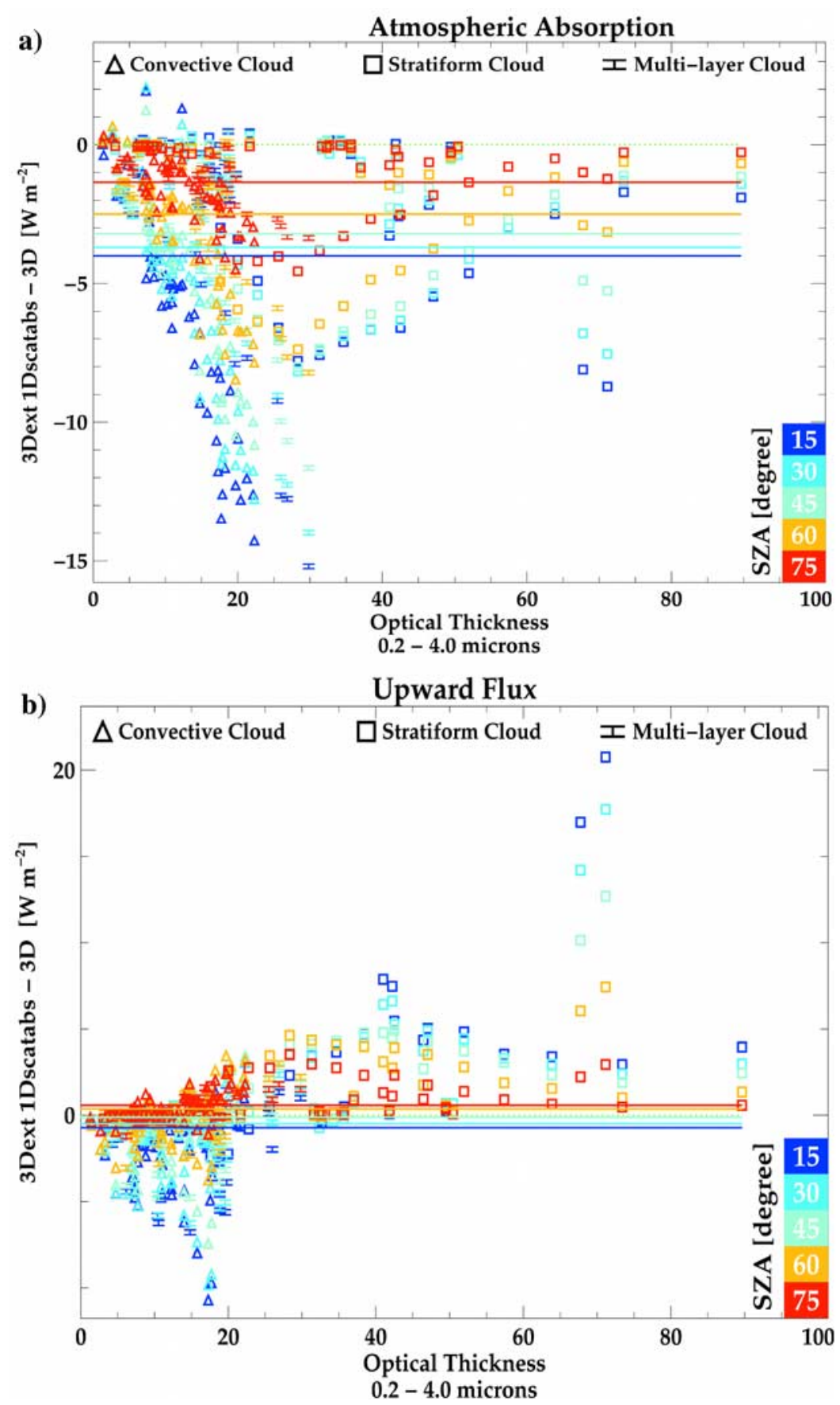

Figure 4. Same as Figure 2 but for the comparison between 3Dext1Dscatabs and full 3-D clouds. See text for details.

(Figure 3a). The large deviations in albedo around $3.8 \mu \mathrm{m}$ of $\sim 3 \%$ are negligible from an energetic point of view (compare Table 2). Relative albedo errors are reduced as the absorptance increases. On the other hand, the spectral distribution of the solar constant leads to largest absolute differences for the upward flux in the spectral range between $0.2 \mu \mathrm{m}$ and $1.185 \mu \mathrm{m}$ with a maximum overestimation of $13 \mathrm{~W} \mathrm{~m}^{-2}$ in the visible spectral range at a SZA of $15^{\circ}$.

[30] The largest errors in atmospheric absorptance (Figure 3d) occur at a SZA of $75^{\circ}$ and at those spectral intervals with largest imaginary parts of the refractive index of water (see Table 2), i.e., largest absorption strength of the hydro- meteors. Water and ice have slightly different spectral refractive indices. However, for the spectral resolution given in Table 2 both phases show essentially the same spectral characteristics. The mean overestimation of $2 \%$ at around $1 \mu \mathrm{m}$ wavelength is more important for the absorbed fluxes than the mean underestimation of $\sim 3 \%$ around $2 \mu \mathrm{m}$ or the mean underestimation of $5 \%$ at $3.8 \mu \mathrm{m}$. No differences are found in nonabsorbing or weakly absorbing spectral regions. In contrast to the upward flux (Figure 3a) the maximum errors in the absorption are shifted toward the IR owing to a compromise between spectral regions of largest irradiance and largest absorptances. Largest under- 


\section{$\operatorname{Abs}(\square+\square)>\operatorname{Abs}(\square+\square)$}

Figure 5. Simple scheme to illustrate a possible mechanism for an enhanced absorption.

estimations reach $\sim 5 \mathrm{~W} \mathrm{~m}^{-2}$ at a SZA of $15^{\circ}$ and $30^{\circ}$. Largest overestimations reach $8 \mathrm{~W} \mathrm{~m}^{-2}$ at $75^{\circ} \mathrm{SZA}$.

[31] As mentioned in the beginning the present study accounts for the full 3-D nature of the radiative transfer problem, i.e., 3-D extinction, absorption, and scattering, whereas most other studies have focused on 3-D extinction fields only. Therefore it is interesting to look at the differences in the radiative transfer results between these two approaches. A similar investigation has been performed by O'Hirok and Gautier [1998, Figure 10] for pure water clouds where it was shown that using a constant effective radius leads to small errors in solar broadband absorption.

[32] We denote the first, complete 3-D scenario by 3Dext3Dscatabs and the latter reduced 3-D scenario with 3Dext1Dscatabs. The term "1Dscatabs" stands for variations in scattering and absorption properties along the vertical direction only. For each horizontal layer, cloud microphysical properties are replaced by their average value. Averaging takes place in cloudy grid cells only, i.e., clear-sky grid cells remain clear sky.

[33] The differences in the solar broadband absorption between these two cloud representations are shown in Figure 4a. The 3Dext1Dscatabs simplification produces a general underestimation of cloud absorption (Figure 4a) up to $15 \mathrm{~W} \mathrm{~m}^{-2}$ at small solar zenith angles, i.e., for the largest solar irradiance, thus accounting for the full 3-D nature of cloud radiative transfer results in an "enhanced absorption" compared to the simplified cloud representation! In fact, O'Hirok and Gautier [1998, Figure 10] also show an underestimation in absorption if the drop sizes are set constant.

[34] Figure $4 \mathrm{~b}$ shows the differences in solar broadband upward flux. Interestingly, the reflected flux is not systematically affected by the horizontal variations in particle size, which makes it difficult to infer cloud absorption from a combination of measurements that include satellite observations.

[35] The physical mechanism for the enhanced absorption is simply the occurrence of larger and thus stronger absorbing cloud particles at the larger optical thicknesses (see Figure 5), i.e., where the number of scattering and absorption events are largest. Homogenizing the absorption strengths horizontally, as it is done in the 3Dext1Dscatabs simplification, leads to both overestimation (in regions of small optical thickness) and underestimation (in regions of large optical thickness) of absorption. Since the regions of large optical thickness have the larger number of scattering events, the underestimation dominates. This simple mechanism significantly reduces absorption compared to the more realistic full 3-D case. Thus from a 3-D radiative transfer point of view, accounting for horizontal variations in cloud microphysical properties may be part of the "missing physics" as proposed in the cloud anomalous absorption debate.

\section{Conclusions}

[36] It is without any doubt that the ICA is a massive improvement in cloud radiative transfer compared to the widely used assumption of plane parallel homogeneous clouds. In fact, recent work has substantially improved the radiation schemes in noncloud-resolving large-scale atmospheric circulation models by applying the ICA to stochastic subgrid parameterizations of cloudiness [Bäuml, 2002]. However, the neglect of horizontal photon transports leads to systematic errors in the ICA and the present work tries to quantify these errors and to explain their origin.

[37] We have shown that the ICA underestimated and overestimated the solar broadband reflected and absorbed flux by as much as $30 \mathrm{~W} \mathrm{~m}^{-2}$ for specific cloud realizations and at specific solar zenith angles. Even when averaged over all clouds, several $\mathrm{W} \mathrm{m}^{-2}$ error remain. This uncertainty is expected to increase with spatial resolution since the ICA assumes an infinite pixel expansion. Therefore it must be concluded that the ICA, despite being a substantial improvement, has its limits and cannot be pushed to arbitrary high accuracies. Consequently, we propose that future cloud radiation parameterization should be based on parameterizations where explicit 3-D cloud radiative transfer calculations have been taken into account to establish the link between domain average cloud and domain average radiation properties.

[38] It should be mentioned that the here performed averaging over cloud realizations is only a rough estimate as the probability density distribution of cloud optical thickness distribution is not flat but skewed to small optical thicknesses. Since the ICA errors are largest at optical thickness values in the range of 10 to 20 , it is to be expected that cloud-averaged ICA errors are larger than those shown here. Therefore it may be interesting to increase the number of cloud realizations to obtain a better coverage of real occurring clouds from which a more realistic averaging can be performed.

[39] Finally, we find it quite interesting to note that if the full 3-D variability of cloud particle absorption is not accounted for, the solar broadband cloud absorption is underestimated by as much as $15 \mathrm{~W} \mathrm{~m}^{-2}$. A simple mechanism has been proposed that significantly enhances absorption compared to simplified cloud radiative transfer simulations and may be part of the "missing physics" that has been proposed in the cloud anomalous absorption debate.

[40] Acknowledgments. The work of R. Scheirer was supported by the GKSS Hochschulprogramm 5.T4.00 G01-HS-1. We are grateful to Lüder von Bremen for providing us with the GESIMA cloud scenarios.

\section{References}

Barker, H. W., Estimating cloud field albedo using one-dimensional series of optical depth, J. Atmos. Sci., 53, 2826-2837, 1996.

Barker, H. W., G. L. Stephens, and Q. Fu, The sensitivity of domain averaged solar fluxes to assumptions about cloud geometry, $Q$. J. R. Meteorol. Soc., 125, 2127-2152, 1999.

Bäuml, G., Influence of the sub-grid scale variability of clouds on the solar radiative transfer computations in the ECHAM5 climate model, Ph.D. thesis, Meteorol. Inst. Univ. of Hamburg, Hamburg, Germany, 2002.

Benner, T. C., and K. F. Evans, Three-dimensional solar radiative transfer in small tropical cumulus fields derived from high-resolution imagery, J. Geophys. Res., 106(D14), 14,975-14,984, 2001. 
Bucholtz, A., Rayleigh-scattering calculations for the terrestrial atmosphere, Appl. Opt., 34, 2765-2773, 1995.

Cahalan, R. F., W. Ridgway, W. J. Wiacombe, T. L. Bell, and J. B. Snider, The albedo of fractal stratocumulus clouds, J. Atmos. Sci., 51, 24342455, 1994.

Cess, R. D., et al., Absorption of solar radiation by clouds: Observation versus models, Science, 267, 496-499, 1995.

Chandrasekhar, S., Radiative Transfer, 393 pp., Dover, Mineola, N. Y., 1960.

Eppel, D. P., H. Kapitza, M. Clausen, D. Jacob, W. Koch, W. Levkov, H.-T. Mengelkamp, and N. Werrmann, The non-hydrostatic mesoscale model GESIMA, Part II, Parameterizations and applications, Contrib. Atmos. Phys., 68, 15-41, 1998.

Fu, Q., M. C. Cribb, H. W. Barker, S. K. Krueger, and A. Grossmann, Cloud geometry effects on atmospheric solar absorption, J. Atmos. Sci., 57, 1156-1168, 2000.

Hagedorn, R., Hydrologiebilanz im Geesthachter Simulationsmodell der Atmosphäre (GESIMA) als Test der mikrophysikalischen Parametrisierung, master's thesis, 81 pp., Math.-Naturwiss. Fakultät der ChristianAlbrechts-Univ. zu Kiel, Kiel, Germany, 1996.

Levkov, L., B. Rockel, H. Kapitza, and E. Raschke, 3-D mesoscale numerical studies of cirrus and stratus clouds by their time and space evolution, Contrib. Atmos. Phys., 65, 35-58, 1992.

Macke, A., and M. Grossklaus, Light scattering by nonspherical raindrops: Implications for lidar remote sensing of rainrates, J. Quant. Spectrosc. Radiat. Transfer, 60(3), 355-363, 1998

Macke, A., J. Mueller, and E. Raschke, Single scattering properties of atmospheric ice crystals, J. Atmos. Sci., 53, 2813-2825, 1996.

Macke, A., J. Mueller, K. Nagel, and R. Stuhlmann, A cellular automaton model for cloud formation, in IRS96: Current Problems in Atmospheric Radiation, edited by W. L. Smith and K. Stamnes, pp. 234-237, A. Deepak, Hampton, Va., 1997.
Macke, A., D. L. Mitchell, and L. V. Bremen, Monte Carlo radiative transfer calculations for inhomogeneous mixed phase clouds, Phys. Chem. Earth, Ser. B, 24(3), 237-241, 1999.

Marshak, A., A. Davis, R. F. Cahalan, and W. J. Wiscombe, Bounded cascade models as nonstationary multifractals, Phys. Rev. E, 49, 5569, 1994.

O'Hirok, W., and C. Gautier, A three-dimensional radiative transfer model to investigate the solar radiation within a cloudy atmosphere, Part I: Spatial effects, J. Atmos. Sci., 55, 2162-2179, 1998.

Pilewskie, P., and F. P. J. Valero, Direct observations of excess solar absorption by clouds, Science, 267, 1626-1629, 1995.

Rothman, L. S., et al., The HITRAN database: 1986 edition, Appl. Opt., 26 , 4058-4097, 1987.

Scheirer, R., and A. Macke, Influence of the gaseous atmosphere on solar fluxes of inhomogeneous clouds, Phys. Chem. Earth., Ser. B, 25(2), $73-$ 76, 2000.

Scheirer, R., and A. Macke, On the accuracy of the independent column approximation in calculating the downward fluxes in the UVA, UVB, and PAR spectral ranges, J. Geophys. Res., 106(D13), 14,301-14,312, 2001.

Thekaekara, M. P., Extraterrestrial solar spectrum, 3000-6100 $\mathrm{\AA}$ at $1-\AA$ intervals, Appl. Opt., 13, 518-522, 1974.

von Bremen, L., E. Ruprecht, and A. Macke, Errors in liquid water path retrieval arising from cloud inhomogeneities: The beam-filling effect, Meteorol. Z., 11(1), 13-19, 2002

A. Macke, Department of Ocean Circulation and Climate, Institute for Marine Research, Duesternbrooker Weg 20, D-24105 Kiel, Germany. (amacke@ifm.uni-kiel.de)

R. Scheirer, Institut für Physik der Atmosphäre, German Aerospace Center, Oberpfaffenhofen, D-82234 Wessling, Germany. (ronald.scheirer@ dlr.de) 